\title{
FOCUS - LES PARADOXES DE LA MÉDIATION CULTURELLE DANS LES MUSÉES
}

Aurélie Peyrin

Caisse nationale d'allocations familiales (CNAF) | «Informations sociales »

2012/2 $\mathrm{n}^{\circ} 170$ | pages 62 à 65

ISSN 0046-9459

Article disponible en ligne à l'adresse :

http://www.cairn.info/revue-informations-sociales-2012-2-page-62.htm

\section{Pour citer cet article :}

Aurélie Peyrin, « Focus - Les paradoxes de la médiation culturelle dans les musées », Informations sociales 2012/2 ( $\left.\mathrm{n}^{\circ} 170\right)$, p. 62-65.

Distribution électronique Cairn.info pour Caisse nationale d'allocations familiales (CNAF).

(C) Caisse nationale d'allocations familiales (CNAF). Tous droits réservés pour tous pays.

La reproduction ou représentation de cet article, notamment par photocopie, n'est autorisée que dans les limites des conditions générales d'utilisation du site ou, le cas échéant, des conditions générales de la licence souscrite par votre établissement. Toute autre reproduction ou représentation, en tout ou partie, sous quelque forme et de quelque manière que ce soit, est interdite sauf accord préalable et écrit de l'éditeur, en dehors des cas prévus par la législation en vigueur en France. Il est précisé que son stockage dans une base de données est également interdit. 


\section{Focus}

\section{Les paradoxes de la médiation culturelle dans les musées}

Aurélie Peyrin - sociologue

La médiation dans les musées recouvre un ensemble de services d'accompagnement des visiteurs qui prennent la forme de conférences, visites guidées, ateliers... Ces services peuvent s'appuyer sur des formes d'interactions différenciées selon le public visé (scolaires, personnes déficientes, adultes en difficulté sociale, etc.) et sur des outils pédagogiques variés. Les acteurs de la médiation sont des animateurs, guides ou conférenciers qui travaillent face au public et accueillent les visiteurs dans le musée, ainsi que ceux qui conçoivent et organisent les activités (concepteurs, enseignants-relais, agents de réservation). Le rôle social de la médiation dans les musées - donner des clés d'interprétation dans des domaines aussi variés que l'art, l'histoire, l'ethnologie ou les sciences naturelles - est le mode d'intervention privilégié de l'État-providence culturel (Schnapper, 1995). Si le concept de médiation culturelle est apparu dans les musées à partir de la fin des années 1980, il recouvre un objectif (la démocratisation) et des activités (dont l'emblème est la visite guidée) dont l'origine se trouve au Louvre, en 1920, et qui n'ont cessé de se développer depuis.

\section{Un groupe professionnel peu homogène}

Selon le point de vue que l'on adopte, le groupe professionnel des médiateurs se présente comme homogène ou hétérogène. La majorité des médiateurs de musées sont des femmes, très diplômées (au minimum bac +4 ou 5). L'âge n'est pas un critère discriminant : on peut être médiateur pendant ses études ou juste après, durant la phase d'insertion, comme on peut faire profession de cette activité et travailler pour le même musée pendant dix, vingt ou trente ans. Par ailleurs, la diversité des musées, que ce soit du point de vue de la taille ou de la nature des collections, entraîne une grande variété de conditions de travail. Alors que certains médiateurs, dans les petits musées, sont seuls à prendre en charge la fonction d'accompagnement, d'autres s'inscrivent au sein d'une véritable équipe où s'observe une division du travail entre conception des visites, animation face aux visiteurs et organisation de l'activité (accueil des groupes, réservations téléphoniques). Enfin, le statut des médiateurs se distribue entre une majorité de contrats de vacations, un contingent d'emplois aidés et une fraction minoritaire d'emplois de fonctionnaires (1). L'activité de médiateur est rarement exercée à plein temps. Pour le moment, ces sources d'hétérogénéité entravent la structuration collective du groupe, c'est-à-dire la capacité de ses membres à construire un destin commun et à défendre une position et une expertise propres. Les médiateurs de musées, dont le dénominateur commun est la prise en charge de l'accompagnement des visiteurs, constituent un groupe « de fait » mais ne parviennent pas à faire reconnaître le lien entre cette activité de travail et une entité collective. Cette professionnalisation incertaine se traduit par trois paradoxes caractéristiques de la médiation dans les musées. 


\section{Une fonction ancienne et omniprésente qui n'a pas d'appellation unifiée}

La population des médiateurs culturels observée en 2002 et 2003 est issue de plusieurs mouvements successifs ou parfois parallèles qui ont contribué à façonner le profil du groupe en lui assignant des missions différentes. Les médiateurs sont, d'une part, les descendants des conférenciers et guides des musées nationaux, recrutés parmi les étudiants et diplômés en histoire de I'art pour accompagner les touristes et les amateurs d'art à partir des années 1920. Ils sont également les héritiers de l'ouverture des musées aux masses laborieuses dans les années 1930 (Peyrouzère, 1999). Ils sont, enfin, les successeurs des animateurs et conférenciers recrutés dans les années 1970 par les collectivités territoriales pour proposer des activités adaptées aux enfants dans un cadre socio-éducatif. Plus récemment, les postes de médiateurs ont été mobilisés (dans la culture comme dans d'autres secteurs) comme supports de contrats emplois jeunes, ce qui a contribué à réorienter les musées vers des activités d'utilité sociale (accueil des handicapés par exemple) (2). Chaque nouvelle impulsion met en lumière une mission prioritaire, qui vient s'ajouter aux autres plutôt que les remplacer ; la médiation est ainsi le dernier avatar de l'accompagnement.

Le terme médiateur a pour origine une forte injonction institutionnelle, apparue entre la fin des années 1980 et le début des années 1990. En lien avec les universitaires qui ont développé le concept de médiation culturelle en philosophie (Caillet et Lehalle, 1995), le ministère de la Culture a poussé les musées à employer ce nouveau vocabulaire pour remplacer les termes existants : conférencier, guide, animateur. Mais les enquêtes auprès des médiateurs eux-mêmes montrent que le terme " médiateur » est loin de faire l'unanimité et les plus expérimentés le rejettent souvent car il n'est pas spécifique aux musées, tandis que les plus jeunes s'y recon- naissent plus que dans le terme " animateur». En résumé, les médiateurs estiment qu'aucun des intitulés existants ne permet d'identifier leur métier ; ils sont bien souvent amenés à se présenter en société en explicitant ce qu'ils font : " J'accueille des groupes dans un musée », " Je conduis les visites».

\section{Des compétences relationnelles occultées}

Dans les musées, les médiateurs ont pour rôle de faciliter la rencontre entre « le public » et les collections des musées, des œuvres d'art ou de culture. La difficulté du métier tient moins aux connaissances requises sur les objets qu'à la diversité des publics accueillis et des modalités de rencontre. Les médiateurs peuvent en effet prendre en charge la visite de groupes constitués (dans la majeure partie des cas, il s'agit de groupes scolaires conduits par un enseignant), mais aussi accueillir ensemble des individus adultes, de tous âges et de tous horizons, qui constituent un groupe pour la seule durée de la visite ou, encore, être à la disposition des visiteurs dans les salles. Dans ces quelques exemples, le médiateur est face à un ensemble distinct dont les membres sont chaque fois renouvelés : chaque visite est une expérience.

À cet égard, il est très étonnant de constater que la plupart des médiateurs sont recrutés sur la base de leurs seuls diplômes, sans aucune qualification ni expérience en communication ou pédagogie. Alors qu'il existe des formations de " médiation culturelle » dans les universités, rares sont les médiateurs en poste à en avoir suivi une. L'apprentissage des compétences relationnelles se fait "sur le tas», par la pratique. Ce mode de fonctionnement présente un inconvénient majeur : il tend à masquer l'existence de compétences spécifiques au métier de médiateur, pourtant reconnues comme des capacités professionnelles dans d'autres métiers. Pédagogie, psychologie et techniques de communication sont notamment indispensables pour 
transmettre des connaissances sur les œuvres. Tout se passe comme si, disent les médiateurs des musées, l'échange avec le public se faisait naturellement: " C'est une question de caractère », $\mathrm{d}^{\prime}$ " ouverture », de "sensibilité », $\mathrm{d}^{\prime}$ " écoute », autant de qualités socialement attribuées à la "nature féminine " (3) et qui ne sont donc pas reconnues dans la relation salariale.

\section{Des agents non titulaires de la fonction publique}

Dès 1920, date de la mise en place de services d'accompagnement dans les musées nationaux, I'activité de médiateur a été considérée comme un débouché idéal pour les femmes diplômées de l'École du Louvre et mères de famille (Peyrin, 2008). La fonction de médiateur conserve encore aujourd'hui les caractéristiques d'une activité professionnelle occasionnelle, destinée à apporter un revenu d'appoint à des femmes mariées et mères de famille. En 2001, dans les musées de France, plus des deux tiers des médiateurs avaient un emploi non permanent, et, dans l'immense majorité des cas, à temps partiel. La part des femmes a aujourd'hui régressé dans la profession, puisque les hommes représentent $40 \%$ des médiateurs de musées en France ; la souplesse des vacations n'attire donc pas seulement les mères de famille.

Pour une partie importante des médiateurs, le travail d'accompagnement représente une transition dans un parcours biographique ou encore un complément à vocation principalement alimentaire. C'est par exemple le cas des étudiants et doctorants, pour lesquels le métier de médiateur est un premier emploi, une activité d'attente avec une insertion espérée dans le secteur académique, la conservation ou la réalisation d'expositions. Pour les artistes, être médiateur est un emploi d'appoint. Dans les deux cas, l'investissement dans le métier est distancié : ce sont les études ou la pratique artistique qui constituent le pilier de l'identité professionnelle. À l'inverse, d'autres médiateurs endossent pleinement leur rôle et cherchent à se stabiliser dans le métier. Pour eux, le principal écueil est la précarité des emplois et l'irrégularité du temps de travail, car ils retirent de ce poste des gratifications symboliques importantes et espèrent durer dans le métier.

Cette diversité des engagements dans le travail constitue la dernière entrave à la constitution d'une identité collective : puisque le groupe n'est pas assez homogène pour s'entendre sur des revendications professionnelles, c'est finalement la tutelle institutionnelle qui promeut la professionnalisation de la médiation, à travers des colloques ou de la formation continue.

Les évolutions récentes du milieu muséal n'ont sans doute pas encore atteint leur pleine mesure en ce qui concerne l'accompagnement. La modernisation, la professionnalisation de la gestion des musées et la diversification des fonctions muséales sont des processus engagés depuis une vingtaine d'années environ dans les établissements publics (Ballé et Poulot, 2004). La mission d'éducation et de diffusion des musées a été reconnue pour la première fois en 2002, lors de la publication de la loi relative aux musées de France. Le processus est donc en route et la marge de développement quantitatif et qualitatif pour le groupe professionnel formé par les médiateurs culturels est considérable. 


\section{Notes}

1 - En 2001, 40 \% étaient vacataires, $20 \%$ en emploi aidé et $9 \%$ en CDD. Seulement $30 \%$ étaient fonctionnaires ou en CDI. Ces données sont issues d'une enquête réalisée par la Direction des musées de France en 2001 dans l'ensemble des musées sous sa tutelle. Les vacataires sont payés " à la tâche », ils ne bénéficient pas des congés payés et ne paient pas de cotisations de chômage.

2 - Dans le secteur de l'art contemporain plus précisément, $58 \%$ des intitulés de poste comportent le terme " médiation ", cf. Zoyem, 2004.

3 - La naturalisation des compétences, qui entraîne leur invisibilité dans le rapport de force salarial, est une constante des métiers féminins. Pour plus de détails à ce sujet, voir Peyrin, 2008.

\section{Bibliographie}

- Ballé C. et Poulot D., 2004, Musées en Europe. Une mutation inachevée, Paris, La Documentation française.

- Caillet E. et Lehalle E., 1995, À I'approche du musée, la médiation culturelle, Lyon, Presses universitaires de Lyon, coll. "Muséologies».

- Hély M., 2010, Les métamorphoses du monde associatif, Paris, Presses universitaires de France (Puf), coll. « Le lien social».

- Ion J. et Tricart J.-P., 2003, Les travailleurs sociaux, Paris, La Découverte, coll. "Repères ».

- Peyrin A., 2010, Être médiateur au musée. Sociologie d'un métier en trompe-I'œil, Paris, La Documentation française, coll. " Musées-mondes »; 2008, " Démocratiser la culture. Une profession intellectuelle au féminin », Travail, genre et sociétés, n 19 , p. 65-85.

- Peyrouzère F., 1999, "Les musées en partage. État et musée sous le ministère Jean Zay, (19361939) ", thèse de doctorat, Université Paris 1 Panthéon Sorbonne.

- Schnapper D., 1995, « De l'État-providence à la démocratie culturelle $»$, Commentaire, $n^{\circ} 68$, p. 889-895.

- Zoyem J.-P., 2004, "Les nouveaux servicesemplois jeunes : bilan fin 2003 », Premières synthèses, premières informations, Dares, mai, $\mathrm{n}^{\circ} 20.1$. 